\title{
Pulsation models of O-rich and C-rich long period variables
}

\author{
Michele Trabucchi ${ }^{1, \star}$, Paola Marigo ${ }^{1}$, Josefina Montalbán ${ }^{1}$, Peter R. Wood ${ }^{2}$, and Léo Girardi ${ }^{3}$ \\ ${ }^{1}$ Dipartimento di Fisica e Astronomia "Galileo Galilei”, Università di Padova, Vicolo dell'Osservatorio 3, \\ I-35122 Padova, Italy \\ ${ }^{2}$ Research School of Astronomy and Astrophysics, Australian National University, Canberra, ACT2611, \\ Australia \\ ${ }^{3}$ Astronomical Observatory of Padova - INAF, Vicolo dell'Osservatorio 3, I-35122 Padova, Italy
}

\begin{abstract}
The intrinsic variability of luminous red giants is an observable providing important information about stellar structure and evolution. Our aim is to provide a solid theoretical background to the wide amount of data coming from present and future variability surveys. We present here the first comparison between long period variables in the LMC (from OGLE-III) and our grid of pulsation models computed for a wide range of stellar parameters and input physics.
\end{abstract}

\section{Introduction}

Low to intermediate mass stars experience the Asymptotic Giant Branch (AGB) during their late evolution, undergoing a number of complex and strongly interacting phenomena such as stellar pulsations, mass loss, dust formation, thermal pulses and dredge-up events. Because of their high luminosity they are often used to characterise stellar populations of external galaxies. Their observed variability is currently attributed to pulsation in modes of low radial orders ([6]). Their periods, that strongly depend on the stellar parameters, provide an additional and powerful observational constraint to be matched by theoretical models, allowing to refine our understanding of late stellar evolution. Existing and future large surveys require large grids of pulsation models and their interpretation as a function of stellar properties. We are currently computing such a grid, planned to be made public (Trabucchi et al. 2017, in preparation), and present here the first comparison with observation.

\section{Methods}

We compute a large grid of pulsation models covering stellar parameters characteristic of the thermal pulsing AGB (TP-AGB) using the code of [1]. For each model, the pulsation period of the fundamental mode and four overtone modes are provided, as well as their growth rates (stability criterion).

In addition to the large coverage of stellar parameters, the main improvement with respect to previous work is the update of opacity, that now includes low temperature atomic and molecular data for the specific chemical composition of oxygen- and carbon-rich AGBs. We use combined data from the ÆSOPUS code ([2]) and the Opacity Project ([4]).

^michele.trabucchi@studenti.unipd.it 


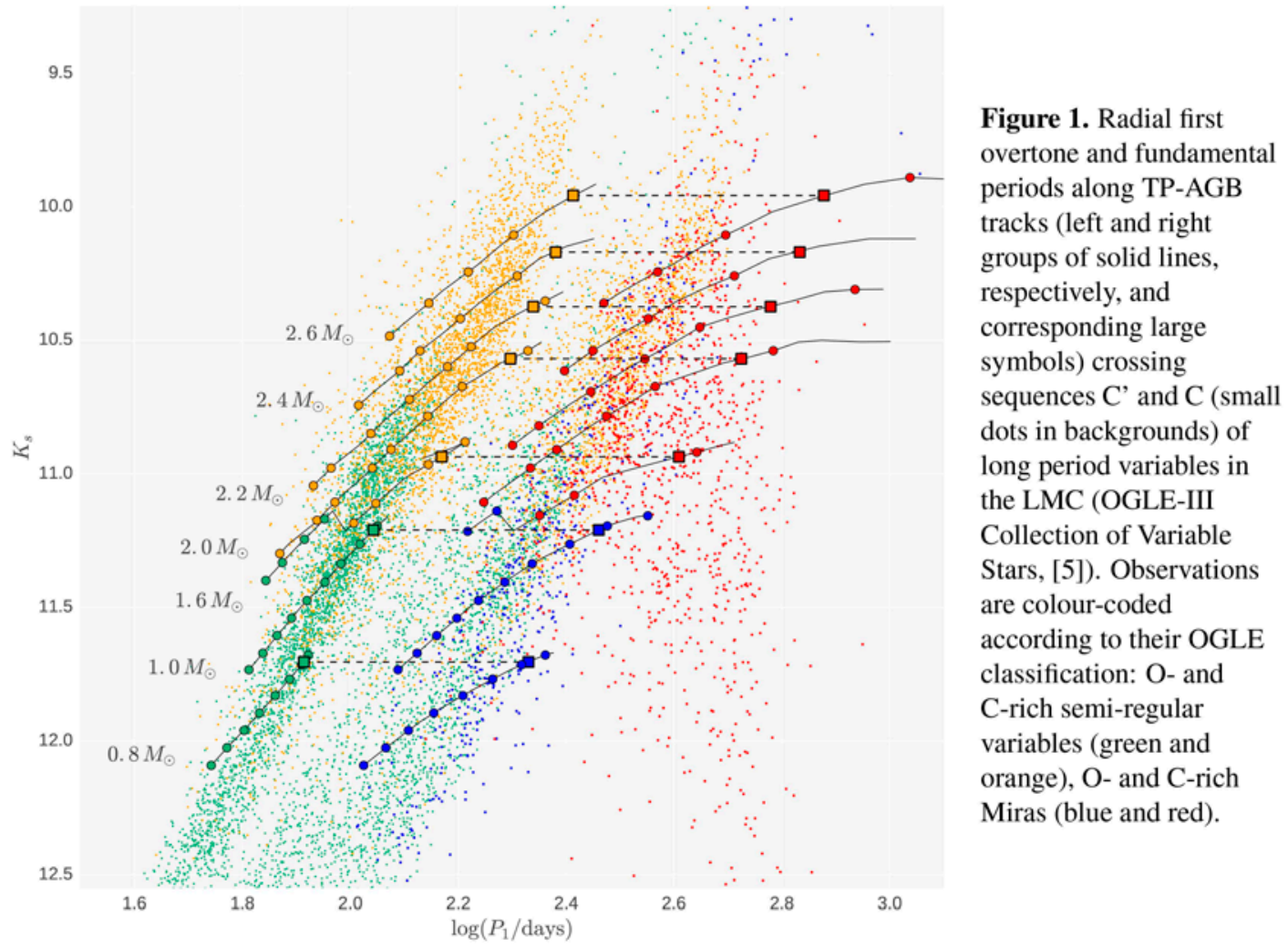

\section{Results}

Here we present the results of pulsation models along TP-AGB evolutionary tracks ([3]) for masses between 0.8 and $2.6 \mathrm{M}_{\odot}$ and metallicity suitable for the LMC, and compare the periods of excited fundamental $(\mathrm{F})$ and first overtone (1O) modes with the period-luminosity (P-L) sequences in the LMC ([5]). In Figure 1 we show the results. We note that: 1) the theoretical and observed properties are in good agreement, including the transition from $\mathrm{O}$ - to C-rich and 2) during TP-AGB evolution, the tracks cross the P-L sequences. Models also predict the luminosity at which the radial order of dominant mode shift downwards, from 10 to $\mathrm{F}$ (square symbols in Fig. 1).

Acknowledgments: We acknowledge the support from the ERC Consolidator Grant funding scheme (project STARKEY, G.A. n. 615604).

\section{References}

[1] Fox, M. W., \& Wood, P. R., ApJ, 259, 198 (1982)

[2] Marigo, P., \& Aringer, B., A\&A, 508, 1539 (2009)

[3] Marigo, P., et al., MNRAS, 434, 488 (2013)

[4] Seaton, M. J., MNRAS, 362, L1 (2005)

[5] Soszyński, I., et al., Acta Astron., 59, 239 (2009)

[6] Wood, P. R., MNRAS, 448, 3829 (2015) 